\section{Case Reports in Ophthalmology}

\title{
Successful Management in a Case of Traumatic Retinal Detachment due to Open Globe Injury Using Microincisional Vitrectomy
}

\author{
Wei-Yu Lai Tsung-Tien Wu \\ Department of Ophthalmology, Kaohsiung Veterans General Hospital, Kaohsiung, and \\ National Yang-Ming University School of Medicine, Kaohsiung, Taiwan
}

\section{Keywords}

Traumatic retinal detachment · Vitrectomy · Open globe injury

\begin{abstract}
Background: Retinal detachment (RD) following ocular trauma often results in guarded visual prognosis and sometimes leads to loss of the eye. With the advent of microincisional vitrectomy surgery and the development of surgical techniques, the management of ocular trauma has been transformed. Case Presentation: A 34-year-old man sustained an open globe injury from fragmented glass at work. He received primary repair and another followup surgery 9 days later, including vitrectomy, silicone oil tamponade, and lensectomy for RD and traumatic cataract at another medical center. However, his retina was totally detached and completely curled up in a roll with choroid on display when he was seen by us 1 month later. He was managed with vigilant and patient peeling and unfolding of the retina using a 23-gauge forceps and silicone oil tamponade, and achieved anatomical success and preservation of his eye at 6 -month follow-up. Conclusions: This report demonstrates that even in cases which appear to be hopeless at presentation, the surgeon's perseverance and surgical technique can salvage an eye that may otherwise be phthisical. It also encourages retinal surgeons to use microincisional vitrectomy to manage severe traumatic RD.
\end{abstract}




\section{Introduction}

Open globe injury remains one of the biggest causes of vision loss. Retinal detachment (RD) occurs in approximately one-third of open globe injuries and is a known risk factor for severe visual impairment [1]. Many studies have demonstrated that RD both at the time of initial presentation and in the postoperative course after open globe repair is associated with poor vision, phthisis, and enucleation. Here we present a case of open globe injury with traumatic RD which appeared seemingly hopeless at presentation but was salvaged after meticulous microincisional vitrectomy.

\section{Case Presentation}

A 34-year-old man sustained a sharp force injury caused by fragmented glass while working, resulting in a penetrating open globe injury in his right eye. He received urgent primary repair on the same day at an outside medical center. However, vitreous hemorrhage with traumatic RD and cataract ensued and warranted a secondary intervention, which included lensectomy and vitrectomy with silicone oil tamponade at the same medical center 9 days later. One month after the trauma, the patient presented to our clinic for further management of his persistent blurred vision. On examination, his visual acuity in the affected eye was hand motion and he was aphakic. Total RD with the retina completely curled up in an egg-roll fashion with retinal rigidity and showing of the choroid layer were found (fig. 1). The patient underwent a three-port 23-gauge pars plana vitrectomy (PPV) along with silicone oil tamponade 1 month after his second surgery. To unfold the retina, the inside out subretinal membrane was meticulously peeled with an intraocular forceps. In order to spread the retina flat open, the edge of the retina was identified. Then, the epiretinal membrane was peeled under perfluorocarbon tamponade. At the temporal lower midperiphery where the jagged retinal pedicle could not be flattened despite thorough membrane removal, relaxing retinectomy was performed. Then gas was injected to displace the subretinal fluid, while the subretinal fluid was drained from the subretinal space with an extrusion needle. Under gas tamponade, a soft-tipped extrusion needle was used to unroll and flatten the retina. Then $20 \%$ perfluoropropane was injected. At 6 months, his retina remained attached and visual acuity had improved to 3/60, without signs of bulbar atrophy (fig. 2).

\section{Conclusions}

Open globe injury is one of the most devastating ocular conditions. Vision loss, chronic pain, disfigurement, and potential loss of the eye are common complications of the disease. Open globe injuries with RD belong to a particular subgroup expected to have an even worse prognosis than those involving the posterior segment without RD. The incidence of traumatic $\mathrm{RD}$ at presentation or within $24 \mathrm{~h}$ after repair ranged from 2.5 to $39.9 \%$ [2]. Attempts at predicting the risk of RD have ben made. Stryjewski et al. [3] retrospectively reviewed patients with open globe injury presenting to the Massachusetts Eye and Ear Infirmary between 1999 and 2011, and used multivariable logistic regression to define the clinical factors associated with RD after open globe injury. They concluded that the presence of vitreous hemorrhage, higher zone of injury, and poorer logarithm of the minimum angle of resolution $(\log M A R)$ visual acuity at the time of presentation after open globe injury were asso- 


\section{Case Reports in Ophthalmology}

Lai and Wu: Successful Management in a Case of Traumatic Retinal Detachment due to Open Globe Injury Using Microincisional Vitrectomy

ciated with RD. A screening tool was thus created: the Retinal Detachment after Open Globe Injury score. Another retrospective chart review of patients with open globe injury who presented to the Ross Eye Institute between September 1, 2009 and March 10, 2014 conducted by Lin et al. [1] showed that patients presenting with poor preoperative visual acuity and vitreous hemorrhage should be regarded as being at high risk for RD, and early intervention should be given.

Urgent surgical intervention to maintain the structural integrity of the eyeball after open globe injury should be attempted at the earliest opportunity. Repair of RD is sometimes performed as a secondary measure. However, the type of surgery, the timing of the intervention, and the type of primary tamponade (gas or silicone oil) remains controversial. There have been reports to support the rationales for vitrectomy performed within 3 days [4], before 2 weeks [5], and between 14 and 30 days [6] after trauma. Because of the retrospective nature of all clinical studies on timing of delayed or early vitrectomy, the current data are of limited value. Salehi-Had et al. [7] reported that secondary intervention performed within 5 weeks after open globe repair had a better visual prognosis than if the secondary surgery was delayed beyond 5 weeks. This difference is likely due to the formation of proliferative vitreoretinopathy (PVR), which forms between 1 and 2 weeks after trauma and exerts tractional forces on the retina [8]. Based on the theory of PVR formation, the authors usually perform secondary repair within 2 weeks after trauma onset. The significant differences that occur with each individual injury make it difficult to independently assess treatment modalities. Ersanli et al. [9] investigated the effect of circumferential scleral buckling on the prognosis of patients undergoing PPV and lensectomy for severe closed globe injury and concluded that scleral buckling may not be necessary for all patients undergoing vitreoretinal surgery for RDs due to closed eye injury. The general anatomical success rate ranged widely. Reed et al. [10] conducted a retrospective, consecutive case series examining patients who had undergone RD repair following zone II and/or III open globe injury repair. The overall rate of final reattachment was 63\% (19 patients), with a mean number of surgeries for RD of 1.5. Rouberol et al. [11] also found a high rate of anatomical success in eyes with open globe injury and RD after PPV repair, with $88 \%$ achieving anatomical success. It is generally agreed that careful cleansing of the vitreous base with the assistance of wide-angle imaging equipment can prevent tractions of the retina. Scleral buckling may help to establish retinal attachment in cases where the base of the vitreous could not be properly cleansed and tractions could not be relieved. The instillation of silicone oil rather than gas as part of the initial repair may help to temporarily confine postoperative PVR to the narrow retro-oil space and thus prevent early postoperative detachment of the macula [12].

Due to the eye stress state after the ocular trauma, congestion, easy bleeding during the surgical procedure, and severe postoperative inflammatory reaction, microincisional vitrectomy is considered preferential. With the development of better instrumentation, microincisional vitrectomy has gained popularity in traumatic cases. The advantages of microincisional vitrectomy include decrease in surgical time and postoperative inflammation as well as maintenance of a stable closed system to avoid swings in intraocular pressure.

Along with the development of surgical technique and microincisional vitrectomy, proper initial surgical management of an open globe injury can create the possibility of salvaging an eye. It is worth highlighting that a surgeon's patience and perseverance also contribute to preserving an eye that may otherwise be lost. Here, we report a case of traumatic RD, seemly hopeless at presentation, who was successfully treated with microincisional vitrectomy. This result should encourage retinal surgeons to use microincisional vitrectomy to manage severe traumatic RD. 
Lai and Wu: Successful Management in a Case of Traumatic Retinal Detachment due to Open Globe Injury Using Microincisional Vitrectomy

\section{Acknowledgment}

This work was supported by Veteran General Hospital Kaohsiung (VGHKS) 103-045.

\section{Authors' Contributions}

W.Y.L. conceived the study, participated in its design and coordination, and drafted the manuscript. T.T.W. participated in the design and coordination of the study as well as the acquisition, analysis, and interpretation of data, and critically revised the manuscript. Both authors read and approved the final manuscript.

\section{Statement of Ethics}

Written informed consent was obtained from the patient for publication of this case report and any accompanying images. A copy of the written consent is available for review by the editor of this journal.

\section{Disclosure Statement}

This study was supported by Kaohsiung Veterans General Hospital, Kaohsiung, Taiwan. The authors have no proprietary or commercial interest in any of the materials discussed in this article.

\section{References}

1 Lin H, Lema GM, Yoganathan P: Prognostic indicators of visual acuity after open globe injury and retinal detachment repair. Retina 2016;36:750-757.

-2 Chee YE, Patel MM, Vavvas DG: Retinal detachment after open-globe injury. Int Ophthalmol Clin 2013;53:79-92.

-3 Stryjewski TP, Andreoli CM, Eliott D: Retinal detachment after open globe injury. Ophthalmology 2014;121:327-333.

4 Coles WH, Haik GM: Vitrectomy in intraocular trauma. Its rationale and its indications and limitations. Arch Ophthalmol 1972;87:621-628.

-5 Vatne HO, Syrdalen P: Vitrectomy in double perforating eye injuries. Acta Ophthalmol 1985;63:552556.

-6 Mieler WF, Mittra RA: The role and timing of pars plana vitrectomy in penetrating ocular trauma. Arch Ophthalmol 1997;115:1191-1192.

7 Salehi-Had H, Andreoli CM, Andreoli MT, Kloek CE, Mukai S: Visual outcomes of vitreoretinal surgery in eyes with severe open-globe injury presenting with no-light-perception vision. Graefes Arch Clin Exp Ophthalmol 2009;247:477-483.

-8 Winthrop SR, Cleary PE, Minckler DS, Ryan SJ: Penetrating eye injuries: a histopathological review. Br J Ophthalmol 1980;64:809-817.

-9 Ersanli D, Sonmez M, Unal M, Gulecek O: Management of retinal detachment due to closed globe injury by pars plana vitrectomy with and without scleral buckling. Retina 2006;26:32-36.

$>10$ Reed DC, Juhn AT, Rayess N, Hsu J, Chiang A: Outcomes of retinal detachment repair after posterior open globe injury. Retina 2016;36:758-763.

$\checkmark 11$ Rouberol F, Denis P, Romanet JP, Chiquet C: Comparative study of 50 early- or late-onset retinal detachments after open or closed globe injury. Retina 2011;31:1143-1149. 


\section{Case Reports in Ophthalmology}

\begin{tabular}{l|l}
\hline DOI: $10.1159 / 000450638$ & $\begin{array}{l}\text { ( } 2016 \text { The Author(s). Published by S. Karger AG, Basel } \\
\text { www.karger.com/cop }\end{array}$ \\
\hline
\end{tabular}

Lai and Wu: Successful Management in a Case of Traumatic Retinal Detachment due to Open Globe Injury Using Microincisional Vitrectomy

\section{Nashed A, Saikia P, Herrmann WA, Gabel VP, Helbig H, Hillenkamp J: The outcome of early surgical repair with vitrectomy and silicone oil in open-globe injuries with retinal detachment. Am J Ophthalmol 2011;151:522-528.}

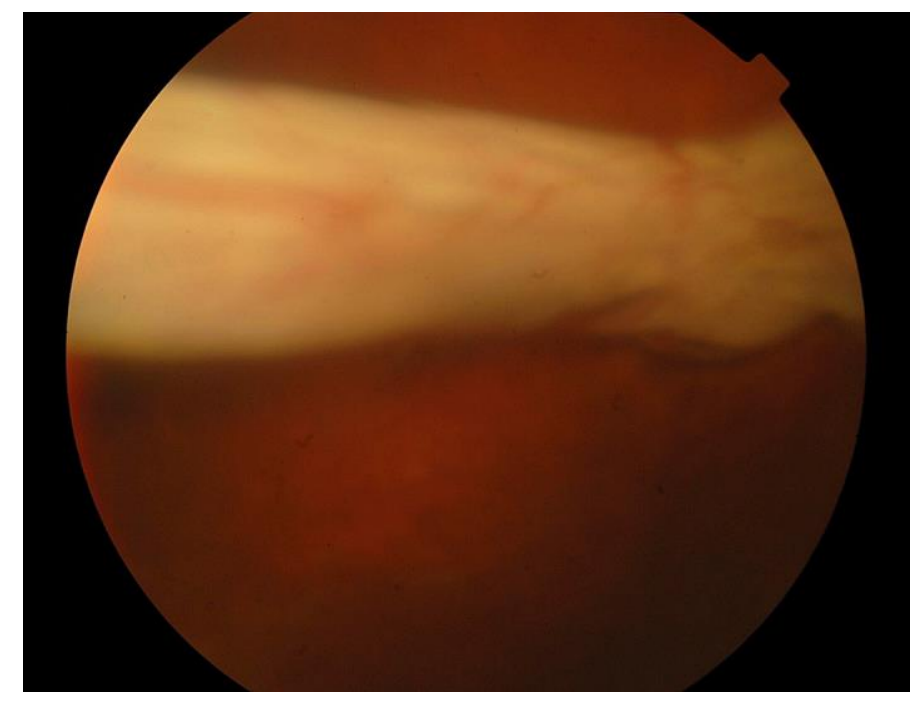

Fig. 1. Total RD with the retina completely curled up in an egg-roll fashion with rigidity and choroid display at initial presentation.

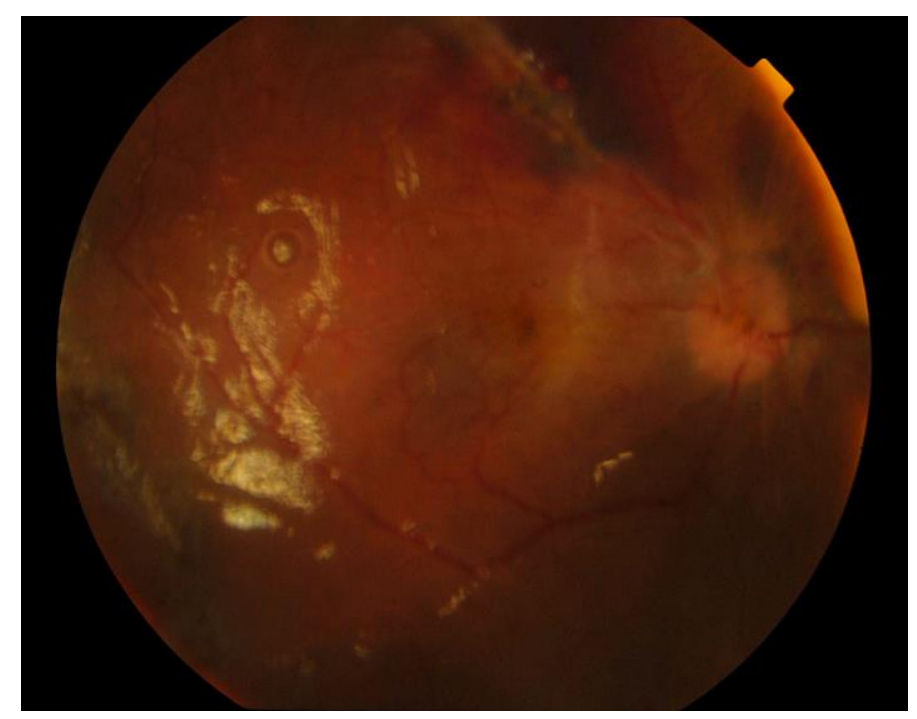

Fig. 2. At 6-month follow-up, the retina remained attached and visual acuity had improved to 3/60, without signs of bulbar atrophy. 